\title{
Bronchial damage and diffuse alveolar hemorrhage following chlorine gas inhalation: A case report
}

\author{
KOSUKE UEMURA ${ }^{1}$, MOMOKO ISONO ${ }^{1}$, KATSUNORI KAGOHASHI ${ }^{2}$, \\ RYUICHI HASEGAWA ${ }^{3}$ and HIROAKI SATOH ${ }^{2}$
}

\author{
Divisions of ${ }^{1}$ General Medicine, ${ }^{2}$ Respiratory Medicine and ${ }^{3}$ Intensive Care Medicine, \\ Mito Medical Center, University of Tsukuba, Mito, Ibaraki 310-0015, Japan
}

Received November 3, 2015; Accepted December 19, 2016

DOI: $10.3892 / \mathrm{etm} .2017 .5161$

\begin{abstract}
Chlorine is a toxic inhalant and sources of exposure for individuals include accidental releases of chlorine vapor due to industrial or chemical transportation accidents. Inhalation of a large quantity of gas may cause circulatory and respiratory disorders or even mortality; however, the effects of a small amount of chlorine gas may be asymptomatic. The present case study presents a successfully treated 55-year-old male patient exposed to chlorine gas, resulting in bronchial damage and diffuse alveolar hemorrhage. Endobronchial and alveolar injuries were evaluated by direct observation using fiberoptic bronchoscopy (FB) and analyzing bronchoalveolar lavage fluid obtained by FB. Taking a precise medical history from the patient is crucial to correctly diagnose toxic gas inhalation. In addition, a timely and proper evaluation with chest imaging as well as FB may provide useful clinical information. Therefore, clinicians should consider performing FB if the circumstances permit.
\end{abstract}

\section{Introduction}

Exposure to chlorine gas may result in serious adverse effects and potentially, patient mortality. Between 2000 and 2005, $\sim 9,000$ cases of chlorine gas exposure were annually reported to poison control centers in the United States (1). Chlorine gas is a potent pulmonary irritant known to cause acute damage in the upper and lower respiratory tracts (2). Chlorine gas inhalation typically occurs following accidental exposure from chemicals used in manufacturing, or in the household and at swimming pools (3). Short-term, high-level exposure due to traffic accidents, chlorine spills or other disasters may result in symptoms of acute airway obstruction occurring, including

Correspondence to: Professor Hiroaki Satoh, Division of Respiratory Medicine, Mito Medical Center, University of Tsukuba, Miya-machi 3-2-7, Mito, Ibaraki 310-0015, Japan

E-mail: hirosato@md.tsukuba.ac.jp

Key words: bronchial damage, diffuse alveolar hemorrhage, chlorine gas inhalation wheezing, cough, chest tightness and dyspnea. More severely affected individuals may suffer from acute lung injury and acute respiratory distress syndrome (2-4) and $\sim 1 \%$ of exposed individuals succumb (4). Mortality occurs primarily due to pulmonary edema with respiratory failure and circulatory collapse (5). Inhaling a large amount of gas may lead to the development of respiratory and circulatory disorders, or even cardiopulmonary arrest (6). Myocardial infarction, acute ischemic stroke and hyperglycemia may be triggered by acute chlorine gas inhalation (5). By contrast, workplace and public exposures are usually long-term, low-level exposures, which may result in increased airway reactivity. It is very difficult to provide a range for asymptomatic exposure as it is related to the materials that generate chlorine gas, the exposure time and the difference in an individual's sensitivity to chlorine gas. An acceptable chlorine concentration is considered to be $<0.5$ parts per million (ppm) and symptoms may appear following exposure to 2-5 ppm chlorine (7). Serious symptoms, including dyspnea, unconsciousness and mortality, usually occur $30 \mathrm{~min}$ to $1 \mathrm{~h}$ following exposure to 30 to $60 \mathrm{ppm}$ chlorine gas. The current study presents the case of a patient exposed to chlorine gas, resulting in bronchial damage and diffuse alveolar hemorrhage, confirmed by fiberoptic bronchoscopy (FB).

\section{Case report}

A 55-year-old male patient brought to the emergency clinic at the Mito Medical Center (Mito, Japan) presented with severe dyspnea. The patient became lethargic and found it impossible to talk due to severe shortness of breath. In addition, impaired consciousness and hypoxia were observed in the patient, who was admitted with a primary diagnosis of acute respiratory failure due to an unknown cause. The vital signs of the patient were measured and recorded, and were as follows: Pulse rate, 190 beats/min (normal range: $\leq 100$ beats/min); blood pressure, $111 / 86 \mathrm{mmHg}$ (within the normal range); respiratory rate, 34 breaths/min (normal range: $\leq 20$ breaths/min); temperature, $37.8^{\circ} \mathrm{C}$ (normal range: $\leq 37.0^{\circ} \mathrm{C}$ ) and oxygen saturation measured by pulse oximetry $\left(\mathrm{SpO}_{2}\right), 82 \%$ (normal range: $\geq 90 \%$ ). Physical examination revealed rigorous inspiratory retraction and coarse crackles. Chest radiograph and computed tomography (CT) scan indicated diffuse ground glass opacity in the left and right lung (Fig. 1). The patient was monitored and 
catheterized. Due to a decrease in $\mathrm{SpO}_{2}$ to $70 \%$ with 10 1/min of oxygen inhalation using a reservoir mask, noninvasive positive pressure ventilation (NPPV) was applied using a Respironics V60 ventilator (Philips Japan, Tokyo, Japan) 14 h following hospital admittance, as $\mathrm{SpO}_{2}$ could not be spontaneously maintained by the patient in this condition. The initial setting of NPPV was continuous positive airway pressure (CPAP) mode with $10 \mathrm{mmHg}$ inhaling $100 \%$ oxygen. Following application of NPPV, $\mathrm{SpO}_{2}$ increased to $90 \%$. CPAP treatment gradually weakened the positive pressure in the airway to atmospheric pressure whilst simultaneously improving $\mathrm{SpO}_{2}$ levels and NPPV was discontinued following $24 \mathrm{~h}$ treatment, and $38 \mathrm{~h}$ after the patient was admitted to hospital. At this point, physicians were made aware that the patient had treated chloric acid and inhaled chlorine gas twice for a few sec $13 \mathrm{~h}$ prior to the arrival at hospital and that dyspnea and bloody sputa began $11 \mathrm{~h}$ prior to patient admittance. FB was performed when the patient was in a conscious state and under local anesthesia (4 $\mathrm{ml} \mathrm{4 \%} \mathrm{lidocaine} \mathrm{delivered} \mathrm{by} \mathrm{bronchoscopic} \mathrm{injection),}$ after the termination of NPPV to identify tracheobronchial appearance and perform bronchoalveolar lavage (BAL) using FB (Olympus BF-260; Olympus Corporation, Tokyo, Japan). Following FB, hyperemic and edematous change was observed in the trachea and bronchus, specifically at interlober spurs between the upper and lower bronchus in the left lung (Fig. 2). BAL fluid from the left lower lobe of the lung had reddish brown appearance with hemosiderin-laden macrophages, suggesting pulmonary hemorrhage. Respiratory condition was maintained using NPPV and oxygen therapy; no corticosteroids or antibiotics were administered to the patient. Diffuse ground glass opacity in the left and right lungs had disappeared on the fifth day of treatment, as indicated by a CT scan. The patient completely recovered and was discharged home five days following hospital admittance with no medication. Once a month for six months, the patient was followed up, but there was no deterioration of respiratory condition.

\section{Discussion}

The mechanism of respiratory tract injury by chlorine gas inhalation is associated with its ability to form hypochlorous and hydrochloric acids in the respiratory tract (8). Due to the relatively low solubility of chlorine gas in water, chlorine is able to reach the periphery of the lungs and cause extensive damage, unlike ammonia and other highly soluble gasses, which are removed from the proximal airway by mucocilliary clearance $(3,8)$. Chlorine gas has a highly irritant effect on the airway epithelium, however; its effect depends on the concentration of inhaled gas as well as the duration of exposure $(6,9)$. A large amount and long duration of chlorine gas inhalation may lead to the development of respiratory and circulatory disorders or even cardiopulmonary arrest, however a small amount and short duration of exposure may be asymptomatic (6). Therefore, chest radiographs and CT scans may not detect any unusual signs, such as pulmonary edema, patchy consolidation, diffuse nodular opacity or vascular congestion (8-10).

Previous case reports have indicated that chlorine gas inhalation may affect various systemic systems other than the respiratory system $(5,6)$. Kose et al $(5)$ documented a

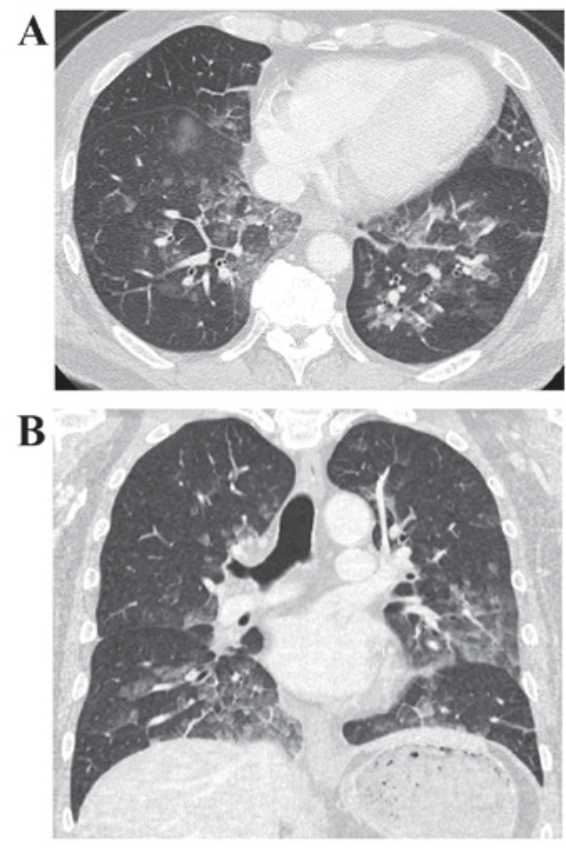

Figure 1. Chest computer tomography scan indicated diffuse ground glass opacity in the lungs. (A) Axial section and (B) coronal section.

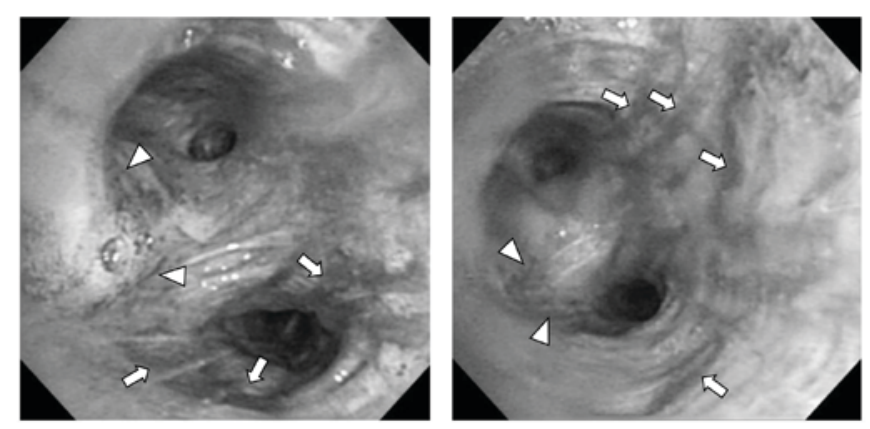

Figure 2. Following fiberoptic bronchoscopy, hyperemic change (arrowheads) and blood attached to the bronchial wall (arrow) were observed at the interlober spur between the upper and lower bronchus in the left lung.

case of acute ischemic stroke, myocardial infarction and hyperglycemia triggered by acute chlorine gas inhalation, and emphasized that physicians should keep in mind all intoxications that may affect various systems and thereby trigger various diseases. However, the patient observed in the present study had a 10-year history of diabetes mellitus and 4-year history of arrhythmia, but no deterioration of these diseases during the clinical course was observed. Li et al (6) reported two cases of pneumomediastinum following acute inhalation of chlorine gas; this complication was not observed in the patient in the present study. Additionally, later complications, such as bronchial asthma, decrease residual volume and increase airway responsiveness have been reported following chlorine gas inhalation (11-14). Therefore, patients require careful follow up observations to detect and prevent the development of such late complications. In the present study, the patient was followed up once a month for six months, however there was no deterioration of respiratory condition. The importance of FB in patients with toxic gas inhalation has been previously 
reported $(9,15,16)$, however performing FB is complicated, due to the severity of the respiratory condition in the majority of patients presenting with symptoms of chlorine gas inhalation. Yarkin et al (9) reported tracheobronchial mucosal injury in a patient treated for chlorine gas inhalation and this was the first reported case to provide FB images. In this patient, hemorrhagic inflammation associated with white necrotic lines on the mucosal surface and disappearance of the cartilage rings of trachea was observed (9). The major bronchial lumens were obstructed with yellowish hard materials thus; FB could not be advanced distally through the main bronchus. Therefore, BAL could not be performed and the patient succumbed to severe acidosis, hypoxemia and high fever on the third day of admission (9). The present study presents a case with FB images as well as BAL findings in a patient with respiratory tract injury due to chlorine gas inhalation. As mentioned previously, there was a difference in the concentration and exposure time of inhaled chlorine gas. Therefore, it seems that there may be a difference in the severity of symptoms experienced by the patient in the study by Yarkin et al (9) and the patient in the current study. To the best of our knowledge, the current case report presents the first successfully treated patient with chlorine gas inhalation, with FB images as well as BAL findings of chlorine gas inhalation.

Yamamoto et al (17) reported two cases of delayed-onset acute lung injury following chlorine gas exposure. Based on the results of Yamamoto et al (17) and other previous reports (18-20), it has been suggested that there may be a latent period lasting up to $10 \mathrm{~h}$ and that symptoms may worsen $48 \mathrm{~h}$ following exposure to chlorine gas. In the current study, the patient developed respiratory failure after $4 \mathrm{~h}$ and symptoms worsened 13-14 h following initial exposure. The application and discontinuation of NPPV occurred 14 and $38 \mathrm{~h}$ following exposure, respectively and FB was performed $40 \mathrm{~h}$ following exposure to chlorine gas. This clinical course suggests that the patient in the present study had 'delayed-onset' lung injury and not 'immediate-onset', as the development and deterioration of symptoms in the patient did not begin rapidly following the inhalation. The amount of chlorine gas inhaled appeared to be small and occurred for a short duration as the patient demonstrated a rapid improvement of respiratory condition without any residual signs or symptoms, despite the diffuse alveolar hemorrhage and endobronchial injury that occurred.

Obtaining a precise medical history is important in order to correctly diagnose patients with toxic gas inhalation. In addition, timely and proper evaluation using chest imaging and FB may provide useful clinical information. Therefore, clinicians should consider performing FB if the circumstances permit. It may be difficult to provide detailed criteria of the appropriate general and respiratory conditions to perform FB safely, as patients may have been exposed to chlorine gas for different durations, inhaled different concentrations of chlorine and respond very differently to chlorine gas inhalation. However, it is important to clarify indications and contraindications of $\mathrm{FB}$ as this may provide clinicopathological information regarding central and peripheral airways in patients that have experienced chlorine gas inhalation.

\section{References}

1. Becker M and Forrester M: Pattern of chlorine gas exposures reported to Texas poison control centers, 2000 through 2005. Tex Med 104: 51-57, 2008.

2. Sexton JD and Pronchik DJ: Chlorine inhalation: The big picture. J Toxicol Clin Toxicol 36: 87-93, 1998.

3. Winder C: The toxicology of chlorine. Environ Res 85: 105-114, 2001.

4. White CW and Martin JG: Chlorine gas inhalation: Human clinical evidence of toxicity and experience in animal models. Proc Am Thorac Soc 7: 257-263, 2010.

5. Kose A, Kose B, Açikalin A, Gunay N and Yildirim C: Myocardial infarction, acute ischemic stroke and hyperglycemia triggered by acute chlorine gas inhalation. Am J Emerg Med 27: 1022.e1-4, 2009.

6. Li B, Jia L, Shao D, Liu H, Nie S, Tang W, Xu B, Hu Z and Sun H: Pneumomediastinum from acute inhalation of chlorine gas in 2 young patients. Am J Emerg Med 29: 357.e1-4, 2011.

7. National Research Council: Emergency and continuous exposure limits for selected airborne contaminants. Vol. 2. Washington, DC: National Academy Press, Committee on Toxicology, Board on Toxicology and Environmental Health Hazards, Commission on Life Sciences, National Research Council, pp5-11, 1984.

8. Kanne JP, Thoongsuwan N, Parimon T and Stern EJ: Trauma cases from Harborview Medical Center. Airway injury after acute chlorine exposure. AJR Am J Roentgenol 186: 232-233, 2006.

9. Yarkın T, Adıgüzel N, Karakurt Z, Güngör G, Aksoy F and Baran R: Chlorine-induced extensive tracheobronchial necrosis concomitantly benzene-induced pancytopenia presented with severe pneumonia. Tuberk Toraks 58: 439-443, 2010.

10. Parimon T, Kanne JP and Pierson DJ: Acute inhalation injury with evidence of diffuse bronchiolitis following chlorine gas exposure at a swimming pool. Respir Care 49: 291-294, 2004.

11. Kilburn KH: Chlorine-induced damage documented by neurophysiological, neuropsychological, and pulmonary testing. Arch Environ Health 55: 31-37, 2000.

12. Gautrin D, Leroyer C, Infante-Rivard C, Ghezzo H, Dufour JG, Girard D and Malo JL: Longitudinal assessment of airway caliber and responsiveness in workers exposed to chlorine. Am J Respir Crit Care Med 160: 1232-1237, 1999.

13. Schwartz DA, Smith DD and Lakshminarayan S: The pulmonary sequelae associated with accidental inhalation of chlorine gas. Chest 97: 820-825, 1990.

14. Donnelly SC and FitzGerald MX: Reactive airways dysfunction syndrome (RADS) due to chlorine gas exposure. Ir J Med Sci 159: 275-277, 1990.

15. Akhavan A, Ajalloueyan M, Ghanei M and Moharamzad Y: Late laryngeal findings in sulfur mustard poisoning. Clin Toxicol (Phila) 47: 142-144, 2009.

16. Cohen MA and Guzzardi LJ: Inhalation of products of combustion. Ann Emerg Med 12: 628, 1983.

17. Yamamoto R, Fujishima S and Ueno K: Two cases of delayed-onset acute lung injury after chlorine gas exposure. JJAAM 20: 390-396, 2009 (In Japanese).

18. Tian X, Tao H, Brisolara J, Chen J, Rando RJ and Hoyle GW: Acute lung injury induced by chlorine inhalation in C57BL/6 and FVB/N mice. Inhal Toxicol 20: 783-793, 2008.

19. Babu RV, Cardenas V and Sharma G: Acute respiratory distress syndrome from chlorine inhalation during a swimming pool accident: A case report and review of the literature. J Intensive Care Med 23: 275-280, 2008.

20. Batchinsky AI, Martini DK, Jordan BS, Dick EJ, Fudge J, Baird CA, Hardin DE and Cancio LC: Acute respiratory distress syndrome secondary to inhalation of chlorine gas in sheep. J Trauma 60: 944-957, 2006. 